\title{
Risk Factors of Stillbirth Among Women Who Gave Birth in Amhara Region Referral Hospitals, Ethiopia, in 2019: A Case-Control Study
}

\author{
Atrsaw Dessie Liyew' \\ Mihretu Molla $\mathbb{D D}^{2}$ \\ Zelalem Nigussie Azene $\mathbb{D}^{3}$ \\ 'Department of Midwifery, College of \\ Health Sciences, Dessie, Ethiopia; \\ ${ }^{2}$ Department of Clinical Midwifery, \\ School of Midwifery, College of Medicine \\ and Health Sciences, University of \\ Gondar, Gondar, Ethiopia; ${ }^{3}$ Department \\ of Women's and Family Health, School of \\ Midwifery, College of Medicine and \\ Health Sciences, University of Gondar, \\ Gondar, Ethiopia
}

Background: Worldwide, 2.6 million stillbirths occur annually and more than three-quarter $\mathrm{s}$ of them are recorded in South Asia and Sub-Saharan Africa. Thus, the aim of this study was to identify risk factors of stillbirth among women who gave birth in Amhara region referral hospitals found in northwest and northcentral, Ethiopia in 2019.

Methods: A multi-center institution-based unmatched case-control study was conducted among 456 mothers who gave birth in Amhara region referral hospitals from October 1 to December 30, 2019. Consecutive and simple random sampling was used to select the cases and controls, respectively. A semi-structured, interviewer-administered questionnaire and patient chart reviews were used to collect the data. Epidemiological (Epi) data version 4.4.2.1 and Statistical Package for the Social Sciences (SPSS) version 25.0 were used for data entry and analysis, respectively. Binary logistic regression was employed. An adjusted odds ratio with a $95 \%$ confidence interval was used to declare statistically significant variables on the basis of $p$-value $<0.05$ in the multivariable binary logistic regression model. Results: Alcohol drinking (adjusted odds ratio $(\mathrm{AOR})=3.02,95 \%$ confidence interval $(\mathrm{CI})$ $=1.24-7.35)$, antepartum hemorrhage $(\mathrm{AOR}=5.74,95 \% \mathrm{CI}=2.67-12.33)$, premature rupture of membrane $(\mathrm{AOR}=2.21,95 \% \quad \mathrm{CI}=1.09-4.44)$, meconium-stained amniotic fluid $(\mathrm{AOR}=8.18,95 \% \mathrm{CI}=4.29-15.60)$, non-use of partograph for labor follow-up $(\mathrm{AOR}=3.89$, $95 \% \mathrm{CI}=2.12-7.17)$, induction of labor $(\mathrm{AOR}=2.12,95 \% \mathrm{CI}=1.09-4.11)$, previous history of stillbirth $(\mathrm{AOR}=2.15,95 \% \quad \mathrm{CI}=1.08-4.26)$, and birthweight less than 2,500 grams (AOR=7.36, 95\% CI=3.43-15.81) increase the odds of stillbirth.

Conclusion: Stillbirth was higher among women who drank alcohol during their pregnancy, experienced antepartum hemorrhage, premature rupture of membrane, meconium-stained amniotic fluid, induction of labor, labor not followed by partograph, previous history of stillbirth, and birthweight less than 2,500 grams. As such, education to stop alcohol drinking during pregnancy, monitoring the progress of labor with partograph, and improving the quality of care for mothers and newborns at the time of pregnancy and childbirth will contribute to preventing stillbirth.

Keywords: risk factors, stillbirth, delivered women, Ethiopia

\section{Background}

Stillbirth is defined as a baby born with no signs of life after a given threshold. The World Health Organization (WHO) defines stillbirth as late fetal death, the death of a baby with birthweight of 1,000 grams or more or a gestational age of 28 completed weeks of age or more or a length of 35 centimeters or more. ${ }^{1}$ 
Every year more than 2.6 million estimated stillbirths occur globally and more than three-quarters of stillbirths occur in South Asia and Sub-Saharan Africa. ${ }^{1}$

According to a population-based multi-country study by the global network in low and middle-income countries from 2010-2013, the overall stillbirth rate was 28.9/1,000 births, ranging from 13.6/1,000 births in Argentina to 56.5/ 1,000 births in Pakistan. ${ }^{1-4}$ In Sub-Saharan Africa, it is approximately 10 -fold higher than that of developed countries (29 vs 3 per 1,000 live births) and more than half of these stillbirths occur during the intrapartum period. ${ }^{1}$

Ethiopia is one of the Sub-Saharan countries which has a high stillbirth rate, ranging from 25.5-33 per 1,000 live births, ${ }^{5,6}$ and in the Amhara region, in particular, the rate of stillbirth was high in 2017 , at 85 per 1,000 live births. $^{7}$

Stillbirth is associated with consequential direct, indirect, psychological, and social costs to the women and their families, society, and governments. These women suffer from anxiety, long-term depression, post-traumatic distress disorder, and stigmatization. ${ }^{8}$

Decreasing the global burden of stillbirth mainly focuses on strategic interventions and to enable these strategic interventions to minimize stillbirth rate, identification of risk factors of stillbirth is needed. ${ }^{9}$ The risk factors of a large proportion of stillbirths remain unknown despite an improvement in antenatal and intrapartum care and extensive testing. ${ }^{10,11}$

Previous studies have identified that several factors were linked with the occurrence of stillbirth. These factors include: advanced maternal age, educational level, grand multiparity, adolescent pregnancy, primiparity, low socioeconomic status, place of delivery, maternal comorbidity (diabetes mellitus, hypertension, HIV infection, preeclampsia), fetal condition (prematurity, low birthweight, post-term pregnancy, congenital anomalies), Antepartum Hemorrhage (APH), prolonged labor, and lack of antenatal care. $^{4,7,12-22}$ However, these factors may vary across countries and time trends depending on the quality and accessibility of care in the health facility and estimates for stillbirth determinants are impeded by various classification systems because of the unavailability of reliable data.

In Ethiopia, a few studies ${ }^{5,7,20,23}$ have been done on risk factors for stillbirth. These studies have involved a single health facility, that does not permit generalization to the wider Amhara region health systems context. The objective of this study was, therefore, to identify risk factors of stillbirth among women who gave birth in
Amhara region referral hospitals in northwest and northcentral Ethiopia.

\section{Methods}

\section{Study Design, Period, and Setting}

A multi-center institution-based unmatched case-control study was conducted from October 1, 2019, to December 30, 2019, in Amhara region referral hospitals, northwestern and northcentral parts of Ethiopia. Amhara region is one of the country's largest regions. It has 12 zones, three-city administrations, and 180 Districts (139 rural and 41 urban). According to the Ethiopian Central Statistics Agency (CSA), the region has a projected population of 21.5 million people. Currently, the region has 80 hospitals (five referrals, two general, and 73 primary), 847 health centers, and 3,342 health posts. ${ }^{24}$ The five referral hospitals are composed of two comprehensive and specialized referral hospitals (University of Gondar comprehensive and specialized referral hospital and Felege Hiwot comprehensive referral hospital) and three referral hospitals (Debre Markos referral hospital, Debre Berhan referral hospital, and Dessie referral hospital). Each hospital serves nearly 5-7 million people, poses $200-400$ beds, and conducts 6,000-8,000 deliveries per year and 16-22 deliveries per day.

\section{Sample Size Determination and Sampling Procedures}

A double population proportion formula was used to determine the sample size by using EPI Info version 7.2 .1 by considering the following assumptions: power (80\%), 95\% Confidence Level (CL), case to control ratio (1:3), 10\% non-response rate, exposure among controls, $(80.1 \%)$, and $\mathrm{COR}=0.48 .{ }^{20} \mathrm{~A}$ total of 468 study participants, with 117 cases and 351 controls, were included in the study. Consecutive sampling was applied to recruit all the cases from each referral hospital until we got the required sample size. The controls (live births) were selected based on the cases among each referral hospital. The number of controls depends on the number of cases obtained from each referral hospital. Accordingly, both cases and controls were taken from Amhara region referral hospitals and the number of controls was 3-times (control-to-case ratio) the number of cases obtained from each referral hospital. A simple random sampling method was applied to select the controls by using the sampling frame of the controls obtained during the occurrence of the event (cases) that 
had happened. The cases were proportionally allocated for each hospital based on the reports of the preceding 3 months of the data collection period.

\section{Study Variables and Measurements}

The occurrence of stillbirth was a response variable, whereas socio-demographic, obstetric factors, maternal behavioral factors, maternal medical factors, and fetal factors were the independent variables of this study. Gestational age measurement was done based on a mother's last menstrual period and ultrasound estimations. Stillbirth was defined as: a baby born $\geq 28$ completed weeks of gestation or weighing $\geq 1,000$ grams or having at least $35 \mathrm{~cm}$ body length, but without any sign of life such as heartbeat, pulsation of the umbilical cord, or definite movement of voluntary muscle. ${ }^{25}$ Live birth was defined as: the complete expulsion or extraction from its mother of a product of conception, irrespective of the duration of the pregnancy, which, after such separation, breathes or shows any other evidence of life, such as beating of the heart, pulsation of the umbilical cord, or definite movement of voluntary muscles, whether or not the umbilical cord has been cut or the placenta is attached. ${ }^{25}$ All women were asked to report their alcohol consumption since pregnancy recognition as there is no safe amount of alcohol during pregnancy. Thus, a woman who drinks any amount of alcohol during pregnancy may be at risk of acquiring fetal anomaly and stillbirth. Premature Rupture of the Membrane (PROM) is usually defined as the rupture of membrane at any time before the onset of uterine contractions but after 28 weeks of gestational age. Premature rupture of membrane which occurs prior to 37 weeks of gestation is referred to as preterm premature rupture of membrane (PPROM), whereas premature rupture of membrane which occurs after 37 weeks of gestation is referred to as term premature rupture of membrane. ${ }^{26}$

\section{Data Collection Tool and Procedures}

Data were collected through a pretested and semistructured questionnaire, a face-to-face interview, and participants' chart review. A total of five data collectors and five supervisors (ie, one BSc midwifery professional for data collection and the case team leader for supervision from each referral hospital) were recruited.

\section{Data Quality Control}

To ensure the quality of data, the questionnaire was first developed in English, then translated into the local language
(Amharic), and finally back into English to check its consistency. Training was given for data collectors and supervisors for a half-day about the purpose of the study and techniques of data collection. Supervisors and the principal investigator closely monitored the day-to-day data collection process. Finally, data were sorted, checked, entered into Epi data, and cleaned (made ready) for analysis. The questionnaire was pre-tested to check the response, language clarity, and appropriateness of the questionnaire while the pretest was done outside the study area at Finote Selam hospital. Depending on the pretest outcomes, corrective measures on language clarity and repetition of words were taken.

\section{Data Processing and Statistical Analysis}

Data cleaning was performed to check for accuracy, completeness, consistencies, and missed values and variables. After the data were checked for completeness and accuracy, data were coded and then entered into Epi data version 4.4.2.1 and exported to Statistical Package for the Social Sciences (SPSS) version 25 for analysis. Descriptive statistics were performed using mean, standard deviation, frequencies, proportions to describe the study population in relation to dependent and independent variables of the study. Multivariable binary logistic regression was used to assess the association between explanatory variables and stillbirth. Variables with a $p$-value $\leq 0.2$ in the bi-variable analysis were considered for the multivariable analysis. In the multivariable binary logistic model, the Adjusted Odds Ratio (AOR) with 95\% CI of the final model were reported to identify the associated factors of stillbirth. Hosmer and Lemeshow goodness of fit test was performed and the decision was made at a $p$-value $>0.05$. Variables having a $p$-value less than 0.05 in the multivariable binary logistic regression models were considered as the significantly associated factors of stillbirth.

\section{Results}

During the 3 months of the study period, 8,550 women were admitted for delivery in Amhara region referral hospitals. Of these, 117 women had intrapartum stillbirths, giving a stillbirth rate of 13.7 per thousand births (Figure 1).

\section{Background Characteristics of the Study Participants}

A total of 468 study participants with 117 cases and 351 controls were included in the study. Of those, $82.9 \%$ of 


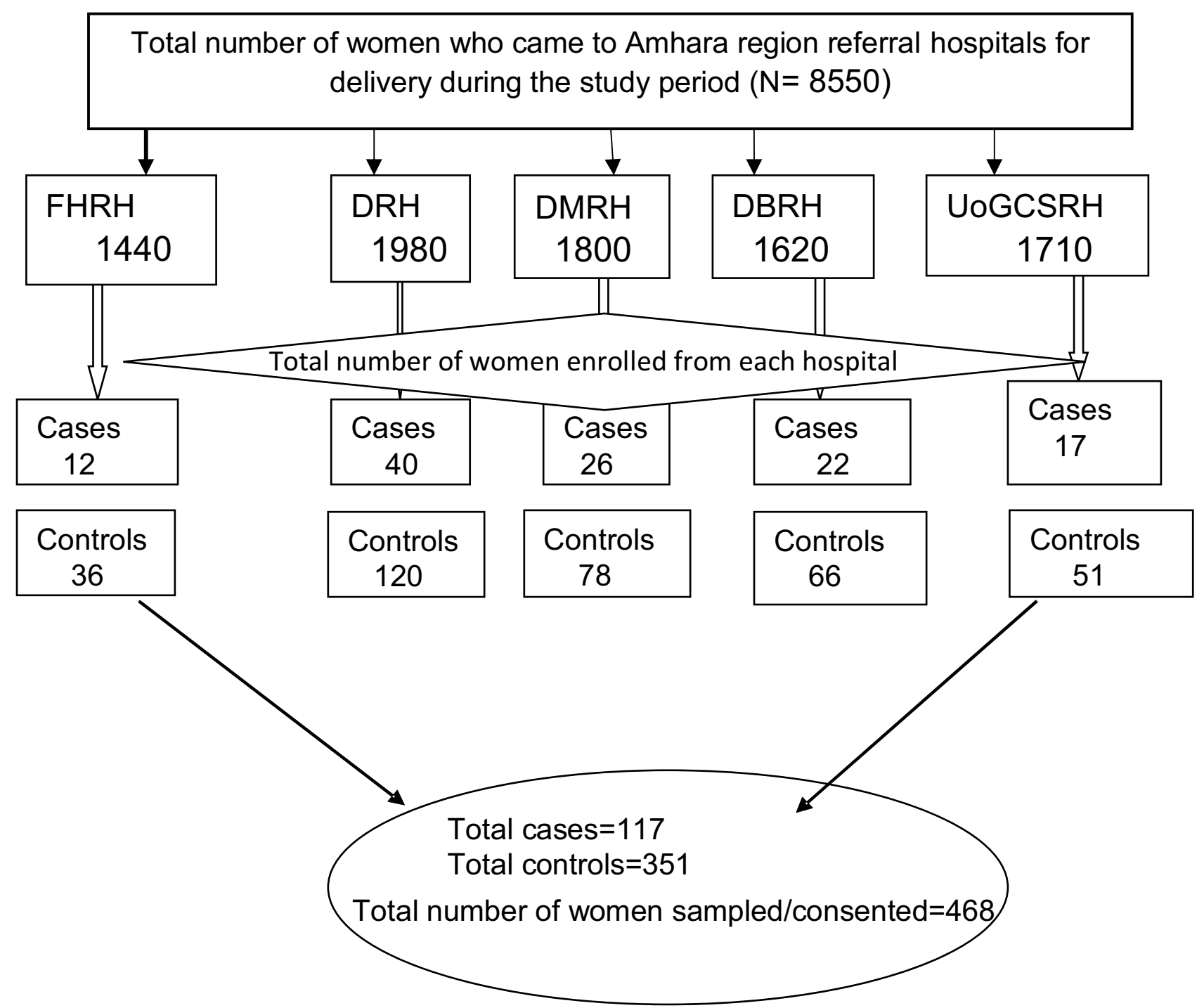

Keys: $\mathrm{FHRH}=$ Felege Hiwot Referral Hospital

DMRH=Debre Markos Referral Hospital

$\mathrm{DBRH}=$ Debre Berhan Referral Hospital

$\mathrm{DRH}=$ Dessie Referral Hospital

UoGCS RH= University of Gondar Comprehensive and Specialized Referral Hospital

Figure I Flow chart of the study population.

cases and $89.1 \%$ of controls were in the age group of 20 34 years.

The mean $( \pm \mathrm{SD})$ age of cases and controls was 27.94 $( \pm 4.83)$ and $28.31( \pm 4.18)$ years, respectively. Regarding the religion of the study participants, $79(67.5 \%)$ of the cases and $213(62.8 \%)$ of controls were Orthodox Christian followers. The cases and controls were statistically significantly different with regard to maternal education, residence, and monthly income (Table 1).
In regards to obstetric complications and medical conditions, about 30 (25.6\%) cases and $22(6.5 \%)$ controls had an antepartum hemorrhage and $28(23.9 \%)$ cases and 50 (14.7\%) controls had a hypertensive disorder of pregnancy. Nearly one-quarter of cases $(22.2 \%)$ and 38 (11.2\%) controls and 48 (41\%) cases and 29 (8.6\%) controls had PROM and meconium-stained amniotic fluid, respectively. Five $(4.3 \%)$ cases and six $(1.8 \%)$ controls and seven $(6 \%)$ cases and $14(4.1 \%)$ controls had diabetes 
Table I Socio-Demographic Profile of Sampled Women in Amhara Region Referral Hospitals, Northwest and Northcentral Ethiopia, in $2019(n=456)$

\begin{tabular}{|c|c|c|c|}
\hline Variables & $\begin{array}{l}\text { Cases } \\
(\mathrm{N}, \%)\end{array}$ & $\begin{array}{l}\text { Controls } \\
(\mathbf{N}, \%)\end{array}$ & p-value* \\
\hline \multicolumn{4}{|l|}{ Age of the mother in years } \\
\hline$<20$ & $5(4.3)$ & $4(1.2)$ & 0.07 \\
\hline $20-34$ & 97 (82.9) & $302(89.1)$ & \\
\hline$\geq 35$ & $15(12.8)$ & $33(9.7)$ & \\
\hline \multicolumn{4}{|l|}{ Religion of the mother } \\
\hline Orthodox & $79(67.5)$ & $213(62.8)$ & 0.62 \\
\hline Muslim & $34(29.1)$ & $110(32.4)$ & \\
\hline Others ${ }^{\mathrm{a}}$ & $4(3.4)$ & $16(4.7)$ & \\
\hline \multicolumn{4}{|c|}{ Marital status of the mother } \\
\hline Single & $8(6.8)$ & $12(3.5)$ & 0.23 \\
\hline Married & $108(92.3)$ & $326(96.2)$ & \\
\hline Others $^{b}$ & $\mathrm{I}(0.9)$ & $\mathrm{I}(0.3)$ & \\
\hline \multicolumn{4}{|l|}{ Ethnicity of the mother } \\
\hline Amhara & III (94.9) & $302(89.1)$ & 0.10 \\
\hline Oromo & $5(4.3)$ & $20(5.9)$ & \\
\hline Others $^{c}$ & I (0.9) & $17(5.0)$ & \\
\hline \multicolumn{4}{|c|}{ Educational status of the mother } \\
\hline Unable to write and read & $40(34.2)$ & $87(25.7)$ & $0.03^{*}$ \\
\hline Primary educations (I-8) & $38(32.5)$ & $89(26.3)$ & \\
\hline Secondary educations (9-12) & $26(22.2)$ & $90(26.5)$ & \\
\hline College and above & $13(11.1)$ & $73(21.5)$ & \\
\hline \multicolumn{4}{|l|}{ Occupation of the mother } \\
\hline Civil servant & $12(10.3)$ & $57(16.8)$ & 0.13 \\
\hline Private employment & II (9.4) & $36(10.6)$ & \\
\hline Housewife & $42(35.9)$ & $107(31.6)$ & \\
\hline Merchant & II (9.4) & $48(14.2)$ & \\
\hline Student & $2(1.7)$ & II (3.2) & \\
\hline Farmer & $39(33.3)$ & $80(23.6)$ & \\
\hline \multicolumn{4}{|l|}{ Residency of the mother } \\
\hline Urban & $65(55.6)$ & $238(70.2)$ & $0.00 *$ \\
\hline Rural & $52(44.4)$ & $101(29.8)$ & \\
\hline \multicolumn{4}{|c|}{ Average monthly income of the family } \\
\hline$<2,500$ ETB & II (9.4) & $14(4.1)$ & $0.0 I^{*}$ \\
\hline $2,500-5,000$ ЕТВ & $92(78.6)$ & $247(72.9)$ & \\
\hline$>5,000$ ETB & $14(12.0)$ & $78(23.0)$ & \\
\hline
\end{tabular}

Notes: Others ${ }^{\mathrm{a}}=$ protestant, catholic, Others $^{\mathrm{b}}$ separated, divorced, Others ${ }^{c}=Q$ manit, Afar. ${ }^{*} p$-value 0.05 considered as a statistically significant difference based on chi-square estimates for the proportion.

Abbreviation: ETB, Ethiopian birr. mellitus and anemia during their pregnancy, respectively (Table 2).

In bi-variable binary logistic regression analysis, maternal education, residence, alcohol drinking during pregnancy, a referral from other health facilities, APH, PROM, the color of amniotic fluid, labor followed by partograph, the onset of labor, previous history of stillbirth, gravidity, and weight at birth were statistically significant with stillbirth at a $p$-value less than 0.2 and considered for multivariable binary logistic regression analysis. After controlling the possible confounders in multivariable analysis, alcohol consumption during pregnancy, APH, PROM, color of amniotic fluid, labor not followed by partograph, induction of labor, previous history of stillbirth, and weight at birth were found to be significantly associated with an increased risk of stillbirth.

In this study, the odds of experiencing stillbirth among mothers who drank alcohol during pregnancy was 3-times (AOR $=3.02,95 \% \mathrm{CI}=1.24-7.35)$ higher than their counterparts. Antepartum hemorrhage was another obstetrics related variable predicting the likelihood of stillbirth. The odds of experiencing stillbirth among mothers who had antepartum hemorrhage was 5.74-times $(\mathrm{AOR}=5.74 \quad 95 \% \mathrm{CI}=2.67$ 12.33) as likely as those who did not have antepartum hemorrhage. Moreover, we found that PROM was an important factor affecting the occurrence of stillbirth. Mothers with PROM had more than 2-times (AOR $=2.21,95 \% \mathrm{CI}=1.09$ 4.44) increased risk of having stillbirth as compared to their counterparts. Mothers who had meconium-stained amniotic fluid during the index pregnancy were 8.18-times (AOR $=8.18,95 \% \mathrm{CI}=4.29-15.60)$ more likely to experience stillbirth than those who had clear amniotic fluid. The onset of labor as part of obstetric related variables was also another factor independently affecting stillbirth. Mothers whose labor started with induction had a 2 -fold $(\mathrm{AOR}=2.12,95 \%$ $\mathrm{CI}=1.09-4.11)$ increased likelihood of experiencing stillbirth as compared with those mothers whose labor started spontaneously. Unsurprisingly, partograph usage at the time of labor was found to be a risk factor for stillbirth. The odds of experiencing stillbirth was 3.89-times (AOR $=3.89,95 \%$ $\mathrm{CI}=2.12-7.17$ ) higher for those mothers whose labor was not followed by partograph than those whose labor was followed by partograph.

The odds of having stillbirth was 2.15-times (AOR=2.15 95\% CI=1.08-4.26) higher among mothers 
Table 2 (Continued).

\begin{tabular}{|c|c|c|c|}
\hline Variables & $\begin{array}{l}\text { Cases } \\
(\mathrm{N} \%)\end{array}$ & $\begin{array}{l}\text { Controls } \\
\text { (N \%) }\end{array}$ & $P$-value \\
\hline \multicolumn{4}{|c|}{ Premature rupture of membrane } \\
\hline Yes & $26(22.2)$ & $38(11.2)$ & 0.003 \\
\hline No & $91(77.8)$ & $301(88.8)$ & \\
\hline \multicolumn{4}{|c|}{ Color of amniotic fluid } \\
\hline Clear & $69(59.0)$ & $310(91.4)$ & $<0.001$ \\
\hline Meconium stained & $48(41.0)$ & $29(8.6)$ & \\
\hline \multicolumn{4}{|c|}{ Labor followed by partograph } \\
\hline Yes & $65(55.6)$ & $298(87.9)$ & $<0.001$ \\
\hline No & $52(44.4)$ & $4 I(12.1)$ & \\
\hline \multicolumn{4}{|l|}{ Obstructed labor } \\
\hline Yes & $12(10.3)$ & $5(1.5)$ & $<0.001$ \\
\hline No & $105(89.7)$ & $334(98.5)$ & \\
\hline \multicolumn{4}{|l|}{ Onset of labor } \\
\hline Spontaneous & $80(68.4)$ & $299(88.2)$ & $<0.001$ \\
\hline Induced & $37(31.6)$ & $40(11.8)$ & \\
\hline \multicolumn{4}{|c|}{ Referred from peripheral health facility } \\
\hline Yes & $77(65.8)$ & $174(5 \mid .3)$ & 0.007 \\
\hline No & $40(34.2)$ & $165(48.7)$ & \\
\hline \multicolumn{4}{|l|}{ ANC follow-up } \\
\hline Yes & $106(90.6)$ & $333(98.2)$ & $<0.001$ \\
\hline No & II (9.4) & $6(1.8)$ & \\
\hline \multicolumn{4}{|c|}{ Number of follow-ups } \\
\hline $\mathrm{I}-2$ & $21(19.8)$ & $4 I(12.3)$ & 0.142 \\
\hline $3-4$ & $64(60.4)$ & $213(64.0)$ & \\
\hline$>4$ & $21(19.8)$ & $79(23.7)$ & \\
\hline \multicolumn{4}{|l|}{ DM in pregnancy } \\
\hline Yes & $5(4.3)$ & $6(1.8)$ & 0.128 \\
\hline No & $112(95.7)$ & $333(98.2)$ & \\
\hline \multicolumn{4}{|l|}{ HIV in pregnancy } \\
\hline Reactive & $9(7.7)$ & $29(8.6)$ & 0.77 \\
\hline Non-reactive & $108(92.3)$ & $310(91.4)$ & \\
\hline \multicolumn{4}{|c|}{ Syphilis screened during pregnancy } \\
\hline Yes & $109(93.2)$ & $300(88.5)$ & 0.17 \\
\hline No & $8(6.8)$ & $39(11.5)$ & \\
\hline \multicolumn{4}{|c|}{ Test result of syphilis } \\
\hline Reactive & $2(1.8)$ & $2(0.7)$ & 0.288 \\
\hline Non-reactive & $107(98.2)$ & $298(99.3)$ & \\
\hline
\end{tabular}

(Continued)
Table 2 Obstetric Characteristics and Medical Conditions of Sampled Women in Amhara Region Referral Hospitals, Northwest and Northcentral, Ethiopia, in $2019(n=456)$

\begin{tabular}{|c|c|c|c|}
\hline Variables & $\begin{array}{l}\text { Cases } \\
(\mathrm{N} \%)\end{array}$ & $\begin{array}{l}\text { Controls } \\
\text { (N \%) }\end{array}$ & $P$-value \\
\hline \multicolumn{4}{|c|}{ APH during current pregnancy } \\
\hline Yes & $30(25.6)$ & $22(6.5)$ & $<0.001$ \\
\hline No & $87(74.4)$ & $317(93.5)$ & \\
\hline \multicolumn{4}{|l|}{ Dx of APH } \\
\hline Placental abruption & $26(86.7)$ & $18(81.8)$ & $<0.001$ \\
\hline Placenta previa & I (3.3) & $4(18.2)$ & \\
\hline Other ${ }^{a}$ & $3(10.0)$ & 0 & \\
\hline \multicolumn{4}{|c|}{ HDP in the current pregnancy } \\
\hline Yes & $28(23.9)$ & $50(14.7)$ & 0.023 \\
\hline No & $89(76.1)$ & $289(85.3)$ & \\
\hline \multicolumn{4}{|l|}{ Type of HDP } \\
\hline Preeclampsia & $21(75.0)$ & $47(94.0)$ & 0.016 \\
\hline Eclampsia & $7(25.0)$ & $3(6.0)$ & \\
\hline \multicolumn{4}{|l|}{ Uterine rupture } \\
\hline Yes & $9(7.7)$ & $\mathrm{I}(0.3)$ & $<0.001$ \\
\hline No & $108(92.3)$ & $338(99.7)$ & \\
\hline \multicolumn{4}{|l|}{ Prolonged labor } \\
\hline Yes & $38(32.5)$ & $99(29.2)$ & 0.505 \\
\hline No & $79(67.5)$ & $240(70.8)$ & \\
\hline \multicolumn{4}{|l|}{ Diagnosis type } \\
\hline $\begin{array}{l}\text { Prolonged latent first stage } \\
\text { of labor }\end{array}$ & $21(55.3)$ & & 0.281 \\
\hline $\begin{array}{l}\text { Protracted/retracted active } \\
\text { phase disorder }\end{array}$ & $5(13.2)$ & $9(9.1)$ & \\
\hline $\begin{array}{l}\text { Prolonged second stage of } \\
\text { labor }\end{array}$ & $12(31.6)$ & $21(2 \mid .2)$ & \\
\hline \multicolumn{4}{|l|}{ Cord prolapse } \\
\hline Yes & $3(2.6)$ & $4(1.2)$ & 0.294 \\
\hline No & II $4(97.4)$ & $335(98.8)$ & \\
\hline \multicolumn{4}{|l|}{ Mode of delivery } \\
\hline Spontaneous vaginal delivery & $87(74.4)$ & $223(65.8)$ & 0.091 \\
\hline Assisted vaginal delivery & $9(7.7)$ & $29(8.6)$ & \\
\hline Cesarian section delivery & $21(17.9)$ & $87(25.7)$ & \\
\hline \multicolumn{4}{|l|}{ Presentation of fetal part } \\
\hline Vertex & $106(90.6)$ & $30 \mathrm{I}(88.8)$ & 0.21 \\
\hline Breech & $5(4.3)$ & $28(8.3)$ & \\
\hline Other ${ }^{\mathrm{b}}$ & $6(5.1)$ & $10(2.9)$ & \\
\hline
\end{tabular}

(Continued) 
Table 2 (Continued).

\begin{tabular}{|l|l|l|l|}
\hline Variables & $\begin{array}{l}\text { Cases } \\
(\mathbf{N} \%)\end{array}$ & $\begin{array}{l}\text { Controls } \\
(\mathbf{N} \%)\end{array}$ & P-value \\
\hline \multicolumn{3}{|l|}{ History of anemia during pregnancy } \\
\hline Yes & $7(6)$ & $14(4.1)$ & 0.41 \\
No & $110(94)$ & $325(95.9)$ & \\
\hline
\end{tabular}

Notes: Other $^{\mathrm{a}}$ local causes, Other ${ }^{\mathrm{b}}$ face and shoulder presentations, $\mathrm{Dx}=$ diagnosis. Abbreviations: APH, antepartum hemorrhage; HDP, hypertensive disorder of pregnancy; ANC, antenatal care; DM, diabetes mellitus; HIV, human immunodeficiency virus.

who had a previous history of stillbirth as compared to their counterparts.

Mothers who gave birth to a newborn whose weight was less than 2,500 grams had a 7-fold (AOR=7.36 95\% $\mathrm{CI}=3.43-15.81)$ increased likelihood of experiencing stillbirth than their counterparts (Table 3).

Maternal educational status, residence, alcohol drinking during pregnancy, a referral from other health facilities, APH, PROM, the color of amniotic fluid, labor followed by partograph, the onset of labor, previous history of stillbirth, gravidity, and weight at birth were independent variables included in the multivariable logistic regression models for which the adjusted odds ratios (AOR) are presented.

Crude odds ratio (COR) is used when we consider one independent and one dependent variable. Adjusted odds ratio (AOR) is used when there was one dependent and more than one independent variable, whereas we want to see an association between one independent and one dependent variable eliminating the effect of other independent variables (confounding factors) in the same case in the same regression model.

\section{Discussion}

The death of the fetus in the last trimester of pregnancy is a source of grief and pain for the families and the communities. To prevent stillbirth, appropriate interventions and strategies are needed. However, to implement the interventions and strategies, identifying the risk factors of stillbirth is of paramount importance. By the same token, we conducted this study to identify the risk factors of stillbirth.

In our study, the odds of having stillbirth among mothers who drank alcohol during pregnancy was 3-times higher than their counterparts. Similar earlier results were also reported in Tete (Mozambique), Tanzania, and Tigray (Ethiopia). ${ }^{13,27,28}$ A plausible explanation is that a woman who drank alcohol while pregnant may harm her developing baby (fetus) because alcohol can pass from the mother's blood into the baby's blood and can damage the baby's cells since alcohol is broken down more slowly in the immature body of the fetus due to the immaturity of the fetal liver. ${ }^{29}$ Several factors can contribute to alcohol consumption during pregnancy; like domestic violence namely, sexual, physical, and emotional/psychological violence, stress, anxiety, depression, marital status, or making local brews as a source of income. ${ }^{30-32}$

Our analysis indicated that having APH during pregnancy was another risk factor that is positively associated with stillbirth. The odds of experiencing stillbirth among mothers who had antepartum hemorrhage was 5.74-times as likely as those who had no antepartum hemorrhage. This result is congruent with other studies conducted earlier in Bangladesh, Nepal, Tanzania, Tete Mozambique, and katsina Nigeria. ${ }^{12,13,19,28,33}$ It is also supported by the results reported in Bonga and Mizan Tepi university teaching hospitals. ${ }^{20}$ This may be owing to the fact that in APH there may be placental separation, acute placental insufficiency, and a decrease in fetal oxygenation and leads to fetal asphyxia which results in intrauterine fetal death. ${ }^{34}$

In the current study, we found that mothers with PROM had more than two times increased risk of having stillbirth as compared to their counterparts. This result is supported by reports from Pakistan, Tanzania, Bahir Dar, and Addis Ababa (Ethiopia). ${ }^{4,28,35,36}$ This may be due to the fact that after rupturing of the membrane, the amniotic fluid becomes oligohydramnios and there may be compression of the umbilical cord and placental abruption which results in fetal asphyxia and ends up with fetal death. An additional explanation could be due to the ascending infection of the uterus (chorioamnionitis) resulting from prolonged premature rupture of the membrane which in turn leads to stillbirth. ${ }^{34}$

The amniotic fluid color was another independent predictor of stillbirth; mothers who had meconium-stained amniotic fluid during the index pregnancy were 8.18times more likely to experience stillbirth than those who had clear amniotic fluid. This finding is similar to previous works in Chandigarh (India), Yemen, and Tigray (Ethiopia). ${ }^{27,37,38}$ The possible reason could be that there may be fetal swallowing of meconium amniotic fluid, resulting in fetal distress and causing fetal death. ${ }^{39}$

In addition, the results of our study showed that onset of labor had a significant association with stillbirth; 
Table 3 Multivariable Regression of Risk Factors of Stillbirth in Amhara Region Referral Hospitals, Northwest and Northcentral Ethiopia, in 2019

\begin{tabular}{|c|c|c|c|c|c|}
\hline \multirow[t]{2}{*}{ Variables } & \multicolumn{2}{|c|}{ Stillbirth } & \multirow[t]{2}{*}{ COR $95 \% \mathrm{Cl}$} & \multirow[t]{2}{*}{ AOR $95 \% \mathrm{Cl}$} & \multirow[t]{2}{*}{$p$-value } \\
\hline & Cases (N) & Controls (N) & & & \\
\hline \multicolumn{6}{|c|}{ Alcohol drinking during pregnancy } \\
\hline Yes & 17 & 16 & $3.43(1.67-7.04)$ & $3.02(1.24-7.35)$ & 0.015 \\
\hline No & 100 & 323 & 1 & I & \\
\hline \multicolumn{6}{|l|}{ APH } \\
\hline Yes & 30 & 22 & $4.97(2.73-9.05)$ & $5.74(2.67-12.33)$ & $<0.001$ \\
\hline No & 87 & 317 & I & 1 & \\
\hline \multicolumn{6}{|c|}{ Premature rupture of membrane } \\
\hline Yes & 26 & 38 & $2.26(1.30-3.93)$ & $2.21(1.09-4.44)$ & 0.027 \\
\hline No & 91 & 301 & 1 & I & \\
\hline \multicolumn{6}{|c|}{ Color of amniotic fluid } \\
\hline Clear & 69 & 310 & 1 & I & \\
\hline Meconium stained & 48 & 29 & $7.44(4.38-12.63)$ & $8.18(4.29-15.60)$ & $<0.001$ \\
\hline \multicolumn{6}{|c|}{ Labor followed by partograph } \\
\hline Yes & 65 & 298 & 1 & 1 & \\
\hline No & 52 & 41 & $5.82(3.56-9.49)$ & $3.89(2.12-7.17)$ & $<0.001$ \\
\hline \multicolumn{6}{|l|}{ Onset of labor } \\
\hline Spontaneous & 80 & 299 & 1 & I & \\
\hline Induced & 37 & 40 & $3.46(2.08-5.76)$ & $2.12(1.09-4.11)$ & 0.025 \\
\hline \multicolumn{6}{|c|}{ Previous history of stillbirth } \\
\hline Yes & 28 & 42 & $2.25(1.32-3.84)$ & $2.15(1.08-4.26)$ & 0.029 \\
\hline No & 88 & 297 & I & I & \\
\hline \multicolumn{6}{|l|}{ Weight at birth } \\
\hline$<2.5 \mathrm{~kg}$ & 30 & 19 & $5.81(3.12-10.81)$ & $7.36(3.43-\mid 5.8 I)$ & $<0.001$ \\
\hline$\geq 2.5 \mathrm{~kg}$ & 87 & 320 & I & I & \\
\hline
\end{tabular}

Abbreviations: APH, antepartum hemorrhage; I, reference group; AOR, adjusted odds ratio; COR, crude odds ratio.

mothers whose labor started with induction had 2-fold increased likelihood of experiencing stillbirth as compared with those mothers whose labor started spontaneously. This finding is similar to prior studies in Chandigarh (India) and in low resource referral hospitals in Zanzibar. ${ }^{37,40}$ The possible explanation could be that during induction of labor there might be an exposure of artificial uterotonic agent and this may result in uterine overstimulation which in turn causes a non-reassuring fetal heart rate and, unless a prompt delivery to prevent stillbirth is performed, the labor may end up with fetal death.
Furthermore, this study yielded that the odds of experiencing stillbirth was 3.89-times higher for those mothers whose labor was not followed by partograph than those whose labor was followed with partograph. This result is in line with studies done in Burkina Faso and Gambia rural hospitals. ${ }^{16,41}$ It is also in accordance with studies carried out in Aksum and Bahir Dar, Ethiopia. ${ }^{36,42}$ The likely explanation for this finding could be the fact that monitoring labor with partograph can help the healthcare provider detect feto-maternal abnormalities as early as possible and take immediate action to save the life of the mother and 
the fetus. However, failure to follow labor with partograph can lead to prolonged labor, which links with stillbirth.

The current study shows that the odds of having stillbirth was 2.15-times higher among mothers who had a previous history of stillbirth as compared with their counterparts. Similar results have been reported in US tertiary care and community hospitals in Bangladesh, Nepal, Haryana, and Kerala (India). ${ }^{12,14,17,33,43}$ This result is also in accordance with a study done in Ethiopia. $^{36}$ The reason might be due to the assumption that the mother might have undiagnosed chromosomal abnormalities that cause intrauterine fetal death. Another possible reason could be the presence of maternal diabetes millets which causes unexplained fetal death. However, this finding contradicts the finding from kersa east Ethiopia. ${ }^{44}$ The hypothesized reason is that women who had previous stillbirth may have been screened and diagnosed in a preconception period for the possible causes of stillbirth and had a regular follow-up to end the pregnancy with a good outcome.

Lastly, the birthweight of newborns had a significant association with stillbirth; mothers who gave birth to a newborn that weighed less than 2,500 grams had a 7-fold increased likelihood of experiencing stillbirth than their counterparts. This finding is supported by similar studies done in Uganda, Nabulus, Tanzania, Nepal, Zimbabwe, and Bonga (Ethiopia). ${ }^{12,20,28,45-47}$ This could possibly be explained by the fact that the presence of intrauterine growth restriction increases the risk of the fetus not getting enough oxygen or other important nutrients. ${ }^{48}$ However, the finding of this study is at variance with the study done in Ghana. ${ }^{49}$ The probable reason for the variation observed may be due to the difference in source population and the study setting.

\section{Strengths and Limitations of the Study}

The main strength of this study is its multi-center design and the involvement of multiple hospitals in Amhara region of Ethiopia. This has allowed its generalization to the wider Amhara region health systems context. The results are not only useful for preventing stillbirths within the participating health facilities; they also have implications for the development of policies for the improvement of maternal healthcare and the reduction of the high rate of stillbirths at a health systems level in the country.

This study also has some important limitations that should be considered when interpreting the results. First, this study did not include the quality of care given in the health facility and other variables that require advanced investigations which may be other risk factors for stillbirth.

Second, due to the retrospective nature of case-control studies, this study may be particularly susceptible to the effects of bias, which may be introduced during the collection of exposure and outcome data. Hence, recall and selection bias can be a threat to our study.

Finally, this study was also unable to ascertain SGA/ growth restriction due to incomplete documentation.

\section{Implications for Policymakers}

The evidence from this finding calls upon policymakers and program managers to play a role in implementing an integrated approach for greater effect on stillbirth prevention through providing reproductive health planning and preconception care, high-quality antenatal care, and an intrapartum care package within the continuum of care for women and newborns including health education and empowerment, detection and management of fetal growth restriction, screening and monitoring in pregnancy to identify high-risk pregnancies so that they can provide more targeted and appropriate treatment and follow-up care, and to monitor fetal wellbeing.

\section{Conclusion}

The study revealed that the likelihood of experiencing stillbirth was higher among women with the following conditions and complications: drinking alcohol during pregnancy, women experiencing APH, PROM, meconiumstained amniotic fluid, induction of labor, labor not followed by partograph, experiencing previous history of stillbirth, and birthweight less than 2,500 grams.

\section{Abbreviations}

ANC, antenatal care; AOR, adjusted odds ratio; APH, A lante partum hemorrhage; CI, confidence interval; COR, crude odds ratio; DM, diabetes mellitus; EDHS, Ethiopian Demographic Health Survey; HDP, hypertensive disorder of pregnancy; HIV, human immunodeficiency virus; LBW, low birthweight; OR, odds ratio; PROM, premature rupture of membrane; SPSS, Statistical Package for the Social Sciences; WHO, World Health Organization.

\section{Data Sharing Statement}

The dataset analyzed during the current study is available from the corresponding author on reasonable request. 


\section{Ethics Approval and Consent to Participate}

Ethical clearance and approval was obtained from the Institutional Review Board (IRB) of the University of Gondar. A letter of cooperation was obtained from the school of Midwifery department of Women's and Family health and submitted to the hospital chief executive officers (CEO). The purpose of the study was explained to the study participants and verbal informed consent was obtained from each of the study participants. Verbal informed consent was approved by the Institutional Review Board of University of Gondar, and this study was conducted in accordance with the Declaration of Helsinki. Reassurance was given for those cases before data collection. No personal identifiers were included in the datasheet and the data taken from the participants were kept confidential and used only for the study purposes.

\section{Acknowledgments}

We are highly indebted to Amhara regional state health bureau for permitting us to conduct the study and providing the necessary preliminary information while conducting this study. We would also like to extend our appreciation to the study participants, supervisors, and data collectors.

\section{Author Contributions}

All authors contributed to the data analysis, drafting or revising the article, have agreed on the journal to which the article will be submitted, gave final approval of the version to be published, and agree to be accountable for all aspects of the work.

\section{Funding}

The authors received no specific funding for this work.

\section{Disclosure}

The authors have declared that they have no conflicts of interest for this work.

\section{References}

1. GBD 2015 Child Mortality Collaborators. Global, regional, national, and selected subnational levels of stillbirths, neonatal, infant, and under-5 mortality, 1980-2015: a systematic analysis for the Global Burden of Disease Study 2015. Lancet. 2016;388(10053):1725-1774. doi:10.1016/S0140-6736(16)31575-6. Erratum in: Lancet. 2017;389 (10064):e1.

2. Saleem S, Tikmani SS, McClure EM, et al. Trends and determinants of stillbirth in developing countries: results from the global network's population-based birth registry. Reprod Health. 2018;15(Suppl 1):100. doi:10.1186/s12978-018-0526-3
3. McClure EM, Saleem S, Goudar SS, et al. Stillbirth rates in low-middle income countries 2010-2013: a population-based, multi-country study from the Global Network. Reprod Health. 2015;12(Suppl 2):S7. doi:10.1186/1742-4755-12-S2-S7

4. Tikmani SS, Zahid N. Rate and Risk Factors of Stillbirth in Pakistan: a Systematic Review. J Pediatr Child Nutr. 2016;2(3):100116.

5. Berhie KA, Gebresilassie HG. Logistic regression analysis on the determinants of stillbirth in Ethiopia. Maternal Health, Neonatol Perinatol. 2016;2(1):10. doi:10.1186/s40748-016-0038-5

6. ICF CSACEa. Ethiopia Demographic and Health Survey 2016. Addis Ababa, Ethiopia, and Rockville, Maryland, USA: CSA and ICF; 2016.

7. Lakew D, Tesfaye D, Mekonnen H. Determinants of stillbirth among women deliveries at Amhara region, Ethiopia. BMC Pregnancy Childbirth. 2017;17(1):375. doi:10.1186/s12884-017-1573-4

8. Heazell AE, Siassakos D, Blencowe H, et al. Stillbirths: economic and psychosocial consequences. Lancet (London, England). 2016;387 (10018):604-616. doi:10.1016/S0140-6736(15)00836-3

9. Wojcieszek AM, Shepherd E, Middleton P, et al. Interventions for investigating and identifying the causes of stillbirth. Cochrane Database Syst Rev. 2018; 4. doi:10.1002/14651858.CD012504.pub2

10. Okochi OD, Isah AD, Agida TE, et al. Stillbirth at a Nigerian Tertiary Hospital. Open J Obstet Gynecol. 2018;8(08):756. doi:10.4236/ ojog.2018.88079

11. Heazell AEP. Stillbirth - a challenge for the 21st century. BMC Pregnancy Childbirth. 2016;16(1):388. doi:10.1186/s12884-0161181-8

12. Ashish K, Nelin V, Wrammert J, et al. Risk factors for antepartum stillbirth: a case-control study in Nepal. BMC Pregnancy Childbirth. 2015;15(1):146. doi:10.1186/s12884-015-0567-3

13. Geelhoed D, Stokx J, Mariano X, et al. Risk factors for stillbirths in Tete, Mozambique. Int J Gynecol Obstet. 2015;130(2):148-152. doi:10.1016/j.ijgo.2015.03.027

14. Lakshmi ST, Thankam U, Jagadhamma P, et al. Risk factors for still birth: a hospital based case control study. Int J Rep, Contraception, Obstet Gynecol. 2017;6(3):970-974. doi:10.18203/2320-1770. ijrcog20170567

15. Lawn JE, Blencowe H, Waiswa P, et al. Stillbirths: rates, risk factors, and acceleration towards 2030. Lancet. 2016;387(10018):587-603. doi:10.1016/S0140-6736(15)00837-5

16. Millogo T, Ouédraogo GH, Baguiya A, et al. Factors associated with fresh stillbirths: a hospital-based, matched, case-control study in Burkina Faso. Int J Gynecol Obstet. 2016;135(S1). doi:10.1016/j. ijgo.2016.08.012.

17. Neogi SB, Negandhi P, Chopra S, et al. Risk Factors for Stillbirth: findings from a Population-Based Case-Control Study, H aryana, I ndia. Paediatr Perinat Epidemiol. 2016;30(1):56-66. doi:10.1111/ ppe. 12246

18. Shrestha J, Shrestha R, Gurung S. Stillbirths-Determining the associated factors and causes according to relevant condition at death: an experience from Pokhara, Nepal. J Nobel Med College. 6(2):58-65. doi:10.3126/jonmc.v6i2.19572.

19. Suleiman BM, Ibrahim H, Abdulkarim N. Determinants of stillbirths in Katsina, Nigeria: a hospital-based study. Pediatr Rep. 2015;7(1). doi: $10.4081 /$ pr.2015.5615

20. Welegebriel TK, Dadi TL, Mihrete KM. Determinants of stillbirth in Bonga General and Mizan Tepi University Teaching Hospitals southwestern Ethiopia, 2016: a case-control study. BMC Res Notes. 2017;10(1):713. doi:10.1186/s13104-017-3058-y

21. Yakoob MY, Lawn JE, Darmstadt GL, et al. Stillbirths: epidemiology, evidence, and priorities for action. Sem Perinatol. 2010;34 (6):387-394. doi:10.1053/j.semperi.2010.09.010

22. DeMarco N, Twynstra J, Ospina MB, et al. Prevalence of low birth weight, premature birth, and stillbirth among pregnant adolescents in Canada: a systematic review and meta-analysis. $J$ Pediatr Adolesc Gynecol. 2021. doi:10.1016/j.jpag.2021.03.003 
23. Tilahun D, Assefa T. Incidence and determinants of stillbirth among women who gave birth in Jimma University specialized hospital, Ethiopia. Pan Afr Med J. 2017;28:299. doi:10.11604/ pamj.2017.28.299.1269

24. Project. HH. HSFR/HFG End of Project Achievement Highlights Amhara. Rockville, MD: Health Finance and Governance Project, Abt Associates; 2018.

25. World Health Organization. Neonatal and Perinatal Mortality: Country, Regional and Global Estimates. World Health Organization; 2006.

26. Gibbs RS, Karlan BY, Haney AF, et al. Danforth's Obstetrics and Gynecology. Philadelphia, PA: Lippincott Williams \& Wilkins; 2008.

27. Tasew H, Zemicheal M, Teklay G, et al. Risk factors of stillbirth among mothers delivered in public hospitals of Central Zone, Tigray, Ethiopia. Afr Health Sci. 2019;19(2):1930-1937. doi:10.4314/ahs. v19i2.16

28. Chuwa FS, Mwanamsangu AH, Brown BG, et al. Maternal and fetal risk factors for stillbirth in Northern Tanzania: a registry-based retrospective cohort study. PLoS One. 2017;12(8):e0182250. doi:10.1371/ journal.pone. 0182250

29. Ornoy A, Ergaz Z. Alcohol abuse in pregnant women: effects on the fetus and newborn, mode of action and maternal treatment. Int J Environ Res Public Health. 2010;7(2):364-379. doi:10.3390/ ijerph 7020364

30. Leonardson GR, Loudenburg R, Struck J. Factors predictive of alcohol use during pregnancy in three rural states. Behav Brain Functions. 2007;3(1):8. doi:10.1186/1744-9081-3-8

31. Mpelo M, Kibusi SM, Moshi F, et al. Prevalence and factors influencing alcohol use in pregnancy among women attending antenatal care in Dodoma region, Tanzania: a cross-sectional study. J Pregnancy. 2018;2018. doi:10.1155/2018/8580318

32. Seabrook JA, Woods N, Clark A, et al. The association between alcohol outlet accessibility and adverse birth outcomes: a retrospective cohort study. J Neonatal Perinatal Med. 2018;11 (1):71-77. doi:10.3233/NPM-181741

33. Nahar S, Rahman A, Nasreen HE. Factors influencing stillbirth in bangladesh: a case-control study. Paediatr Perinat Epidemiol. 2013;27(2):158-164. doi:10.1111/ppe.12026

34. Gabbe SG, Niebyl JR, Galan HL, et al. Obstetrics: Normal and Problem Pregnancies e-Book. Elsevier Health Sciences; 2016.

35. Sirak B, Mesfin E. Maternal and perinatal outcome of pregnancies with preterm premature rupture of membranes (pprom) at tikur anbessa specialized teaching hospital, Addis ababa, Ethiopia. Ethiop Med J. 2014;52(4):165-172.

36. Worede DT, Dagnew GW. Determinants of stillbirth in Felege-Hiwot comprehensive specialized referral hospital, North-west, Ethiopia, 2019. BMC Res Notes. 2019;12(1):579. doi:10.1186/s13104-0194621-5
37. Newtonraj A, Kaur M, Gupta M, et al. Level, causes, and risk factors of stillbirth: a population-based case control study from Chandigarh, India. BMC Pregnancy Childbirth. 2017;17(1):371. doi:10.1186/ s12884-017-1557-4

38. Obadi M, Taher R, Qayad M, et al. Risk factors of stillbirth in Yemen. $J$ Neonatal Perinatal Med. 2018;11(2):131-136. doi:10.3233/NPM-181746

39. Jacques SM, Qureshi F. Does in utero meconium passage in term stillbirth correlate with autopsy and placental findings of hypoxia or inflammation? J Maternal-Fetal Neonatal Med. 2020;1-7. doi:10.1080/14767058.2020.1770217

40. Maaloe N, Housseine N, Bygbjerg IC, et al. Stillbirths and quality of care during labour at the low resource referral hospital of Zanzibar: a case-control study. BMC Pregnancy Childbirth. 2016;16(1):351. doi:10.1186/s12884-016-1142-2

41. Jammeh A, Vangen S, Sundby J. Stillbirths in rural hospitals in the gambia: a cross-sectional retrospective study. Obstet Gynecol Int. 2010;2010:186867. doi:10.1155/2010/186867

42. Berhe T, Gebreyesus H, Teklay H. Prevalence and determinants of stillbirth among women attended deliveries in Aksum General Hospital: a facility based cross-sectional study. BMC Res Notes. 2019;12(1):368. doi:10.1186/s13104-019-4397-7

43. Group SCRNW. Association between stillbirth and risk factors known at pregnancy confirmation. JAMA. 2011;306(22).

44. Assefa N, Berhane Y, Worku A, et al. The hazard of pregnancy loss and stillbirth among women in Kersa, East Ethiopia: a follow up study. Sexual Reprod Healthcare. 2012;3(3):107-112. doi:10.1016/j. srhc.2012.06.002

45. Moyer CA, Kolars CK, Oppong SA, et al. Predictors of stillbirths and neonatal deaths in rural western Uganda. Int $J$ Gynecol Obstet. 2016;134(2):190-193. doi:10.1016/j.ijgo.2016.01.009

46. Cung TG, Paus AS, Aghbar A, et al. Stillbirths at a hospital in Nablus, 2010: a cohort study. Glob Health Action. 2014;7:25222. doi:10.3402/gha.v7.25222

47. Feresu SA, Harlow SD, Welch K, et al. Incidence of and sociodemographic risk factors for stillbirth, preterm birth and low birthweight among Zimbabwean women. Paediatr Perinat Epidemiol. 2004;18(2):154-163. doi:10.1111/j.1365-3016.2003.00539.x

48. Ashwini Vishalakshi L, Reddi Rani P. Early detection of IUGR: can it prevent stillbirth? Int J Rep, Contraception, Obstet Gynecol. 7 (9):3630

49. Badimsuguru AB, Nyarko KM, Afari EA, et al. Determinants of stillbirths in Northern Ghana: a case control study. Pan Afr Med J. 2016;25(Suppl 1):18. doi:10.11604/pamj.supp.2016.25.1.6168

\section{Publish your work in this journal}

The International Journal of Women's Health is an international, peerreviewed open-access journal publishing original research, reports, editorials, reviews and commentaries on all aspects of women's healthcare including gynecology, obstetrics, and breast cancer. The manuscript management system is completely online and includes a very quick and fair peer-review system, which is all easy to use. Visit http://www.dovepress.com/testimonials.php to read real quotes from published authors. 